\title{
Microsurgery Training for the Twenty-First Century
}

\author{
Simon Richard Myers ${ }^{1}$, Stefan Froschauer ${ }^{2}$, Yelena Akelina ${ }^{3}$, Pierluigi Tos $^{4}$, Jeong Tae Kim ${ }^{5}$, \\ Ali M Ghanem ${ }^{1}$ \\ ${ }^{1}$ Academic Plastic Surgery Group, Barts and The London School of Medicine and Dentistry, London, UK; ${ }^{2}$ Microsurgical Training and \\ Research Centre, Centre for Biomedical Technology and Research, Linz, Austria; ${ }^{3}$ Microsurgical Training and Research Laboratory, Columbia \\ University, New York, USA; ${ }^{4}$ Department of Reconstructive Microsurgery, Orthopaedic and Trauma Centre-CTO, Turin, Italy; Department \\ of Plastic Reconstructive Surgery, Hanyang University Hospital, Seoul, Korea
}

Current educational interventions and training courses in microsurgery are often predicated on theories of skill acquisition and development that follow a 'practice makes perfect' model. Given the changing landscape of surgical training and advances in educational theories related to skill development, research is needed to assess current training tools in microsurgery education and devise alternative methods that would enhance training . Simulation is an increasingly important tool for educators because, whilst facilitating improved technical proficiency, it provides a way to reduce risks to both trainees and patients. The International Microsurgery Simulation Society has been founded in 2012 in order to consolidate the global effort in promoting excellence in microsurgical training. The society's aim to achieve standarisation of microsurgical training worldwide could be realised through the development of evidence based educational interventions and sharing best practices.

\section{Keywords Curriculum / Education / Microsurgery / Teaching}

Correspondence: Ali M Ghanem Academic Plastic Surgery Group, Centre for Cutaneous Research, Blizard Institute, Barts and The London School of Medicine and Dentistry, 4 Newark Street, London E1 2AT, UK Tel: +44-77-64-18-44-77 Fax: +44-87-08-38-14-74 E-mail: a.ghanem@qmul.ac.uk

No potential conflict of interest relevant to this article was reported.
Surgical training within all specialties has changed fundamentally within the Western world and beyond in recent years with the pressures of resource limitations and an increasing preoccupation with safety and regulation. Increasingly, the requirement of any trainee by any training body is to gain defined proficiencies and competencies within a defined period. Whilst the training hours per week, and indeed the training years, have often been truncated, the development of objective evidence of skills acquired in training has lagged behind, and any existing measures often remain subjective at best. Moreover, the link between ex- isting measures of competence have seldom been convincingly linked to clinical outcome. Just as in producing evidence for clinical surgical practice through research, the training outcome area is generally considered too multifactorial and complex to address.

Microsurgery is arguably one of the most specialized technical areas of modern surgery, especially now that robotic microsurgery is developing. Whilst microsurgery now engages many specialty areas beyond plastic surgery alone, it has at its roots those key elements of that specialty - innovation and an atten- 
tion to detail. Those elements are now are rapidly being embraced by the microsurgery community to address the challenge of objective measures of skill, of skill acquisition, and of skill maintenance. There are many elements that contribute to a successful clinical outcome from a microsurgery case, but failure of microvascular anastomosis itself will unquestionably result in a failed reconstruction. There is a further advantage to the pivotal role of anastomosis to clinical outcome, in that this element can be manageably broken down into sub-elements that can indeed be analyzed and objectively assessed. The established approach remains "semi-objective" by global rating scoring of those subelements by observers. This is labour intensive and has not become standard; and although other approaches including hand motion analysis have shown promise, they have been slow to progress.

Microsurgery training has embraced the simulation environment from the outset, with training on an in-vivo rat model at some point being a standard of sorts, albeit with no objective output. The ex-vivo simulated environment has become increasingly attractive on a cost basis, and with the aim of limiting animal exploitation. The ex vivo simulated environment offers the further advantage of a cost-effective route to objective outcome measures of skill. It is only in this environment, that such measures can be explored, developed, and validated as transferable to clinical relevance. It is in this environment that learning curves can be established for defined exercises. The methodologies established are likely to prove important to other techniques and specialties as clinical regulation moves into the era of revalidation on both sides of the Atlantic and, very soon, Worldwide.

All these new avenues in microsurgery training have led a group of dedicated microsurgery educators from all over the world to identify the common ground of various educational systems offered globally in different institutions that had been operating independently for decades with hardly any active form of networking or experience sharing.

In a first meeting, an educational session during the National Meeting of the Italian Society for Microsurgery in Palermo in October 2011, more than 10 institutions gave an overview of their work and training systems. The main aim of this meeting was to initiate a network which would facilitate dialogue among those institutions. Analysing the lectures from Palermo, we found rather diverse educational systems, with regard to the training time, tutor/trainee ratio, types of exercises and kinds of simulation training models on offer. All these factors and the growing importance of standardization in these times led to the idea of establishing a modular educational system generally accepted among these institutions to start with, towards a global consensus as a long-term aim.

A second educational session was organised at the Meeting of the European Federation of Societies of Microsurgery in Paris in April 2012. Here, the idea of a step-wise modular standardised training system was discussed extensively. The main topics that were explored in Paris include the necessity of a standardization in microsurgical training systems under the umbrella of the European Microsurgery Research Association (EMRA) and the possibility of multi-centre studies to improve the quality of education.

These efforts led to the foundation of the International Microsurgery Simulation Society (IMSS) in the following months and its inaugural meeting on June 30, 2012 at Barts and the London School of Medicine and Dentistry, UK, chaired by Professor Simon Myers.

In an outstandingly organised meeting at Mile End Campus of Queen Mary University of London, representatives of 20 educational institutions from 9 countries of Europe, the United States, and Asia agreed on the aims of the newly founded society: Quality Improvement and Innovation in Microsurgery Education.

Since the establishment of the IMSS, interest in the standardization of microsurgical training and education and the establishment of a modular system have grown in the international arena with initiatives emerging from the International Society for Experimental Microsurgery (ISEM) and the American Society for Reconstructive Microsurgery (ASRM). These initiatives are currently being crystallised and will be discussed at the next meeting of the IMSS on May 11, 2013 in New York, USA, hosted by Columbia University and shortly afterwards on a larger scale when these detailed concepts will be presented to the World Society of Reconstructive Microsurgery (WRSM) in Chicago, USA this July.

The Archives of Plastic Surgery (APS) Editor-in-Chief and the Korean Society of Microsurgery have been very interested in these important developments in microsurgery education. APS has published several articles that lay the foundations for this timely international dialogue towards quality improvement and innovation in microsurgery education, which will no doubt bring us, and more importantly our patients and students, to a new era of enhanced learning and training. In the end, our goal is better outcomes and finer artistry in reconstructive microsurgery to the benefit of our patients, our true and best educators always. 